\title{
Structured Estimation of Tire Forces and the Ground Slope Using SM Observers
}

\author{
Belgacem Jaballah Nacer Kouider M'Sirdi \\ Laboratory of the Sciences of the Information and the Systems, LSIS CNRS UMR 7296, Domaine Universitaire St Jerome, Avenue Escadrille \\ Normandie Niemen, 13397 Marseille, France
}

\begin{abstract}
In agricultural context, the principal cause of serious accidents for all-terrain vehicles (ATVs) is rollover. The most important parameters related to this risk is the ground slope. In this paper, we propose a structured observer to estimate the system states and the longitudinal tire forces using only wheel angular velocities measurement. The robust estimation is based on a second order sliding mode observer. This estimation is then used to build up a ground slope estimation. The algorithm is composed by two cascaded estimators. This structured estimation is then applied to the model of an agricultural vehicle G7 (Gregoire ${ }^{\mathrm{TM}}$ ) integrated in the driving simulation environment $\mathrm{SCANeR}^{\mathrm{TM}}$-Studio.
\end{abstract}

Keywords: All-terrain vehicles, rollover situation, sliding modes observers, longitudinal tire forces, ground slope.

\section{Introduction}

All-terrain vehicles (ATVs) are frequently used in the agricultural context. Yet despite their great contribution to the productivity of the agricultural industry, the ATVs are associated with a large number of dangerous accidents. Their geometric characteristics and heaviness increase the risk of unsafe or unstable situations. There are a number of risk factors associated to the ATVs accidents. Over $90 \%$ of the accidents occurred while driving on ground slope and two thirds of the accidents resulted in the ATVs rolling over ${ }^{[1,2]}$. Then it is extremely important to estimate the tire contact forces, the velocities and the ground slope.

In the literature, many studies deal with observers for contact tire forces. The objective may be comfort analysis, design or increase in safety by means of enhancing the controllability of the vehicle on the ground. In [3], Ono et al. estimates friction force characteristics using extended braking stiffness (XBS) method by applying online leastsquares method. This method is also applied in [4]. Kiencke and Nielsen ${ }^{[5]}$ presents a procedure for real-time estimation of adhesion $\mu$. He develops a relation between $\mu$ and the wheel slip ratio $s$. In $[6,7]$, Ray estimates the forces on the tires with an extended Kalman filter. Villagra et al. ${ }^{[8]}$ presents an estimation of the road maximum adherence $\mu$ based an algebraic approach using ALIEN algorithms. In [9], Gustafsson derives a scheme to identify different classes of roads. He assumes that by combining the slip and the initial slope of the road adhesion curve, it is possible to distinguish between different road surfaces. Recently, many studies have been performed on estimation of the frictions and contact forces between tires and road ${ }^{[10,11]}$. However, these parameters are difficult to get and not easy to measure. There are some difficulties to have them in real time. In [12], the authors deal with the simultaneous estimation of states and unknown inputs for a class of Lipschitz nonlinear systems using only the measured outputs. A two-

\footnotetext{
Manuscript received January 29, 2013; revised July 18, 2013

This work was supported by French National Research Agency (Projet ANR: ActiSurTT, LSIS 2013).
}

step approach to closed-loop identification of the polynomial $\mathrm{NARX}^{1} / \mathrm{NARMAX}^{2}$ systems with variable structure control (VSC) is presented in [13]. In [14], an observerbased nonlinear control method has been developed and implemented to provide accurate tracking control of a limited angle torque motor.

Wheel-soil interactions in ATVs are very complex and make the forces estimation problem more difficult than that for vehicles on roads. We should take into account that both the wheel and the soil, are deformable bodies. The main objective here is to ensure an accurate estimation of the contact forces for a better diagnosis of rollovers for all-terrain vehicles. Moreover, the wheel's sinking in the ground generates resistive forces that might influence the vehicle's dynamics. A main reason that could cause an all-terrain vehicle crash often comes from the ground slope. Contact forces (wheel-soil interaction) are important factors in determining the vehicle's dynamics and safety.

In [15], we have proposed two sliding mode (SM) observers (first and second order) to estimate the longitudinal tire forces using only wheel's angular positions measurement. This observer is developed for light weight vehicles. In [16], the convergence proof is detailed for light weight vehicles on roads. For all-terrain vehicle, the problem is more difficult.

Then the main contribution here is to develop two sliding mode cascaded observers to estimate both the system state and the longitudinal tire forces $F_{x}$ for all-terrain vehicles and the ground slope.

The vehicle, considered here, is an all-terrain heavy tractor (G7.240 carrier) rolling off road on a deformable ground. The vehicle and the environment are clearly different from those considered in our previous papers where the position measurements are assumed available. In this case, the velocities are measured and a finite time converging observer provides state estimates. This allows to get good condition to estimate the relative ground slope.

\footnotetext{
${ }^{1}$ Nonlinear autoregressive exogenous model.

${ }^{2}$ Nonlinear autoregressive moving average model with exogenous inputs.
} 
This paper is organized as follows. Section 2 presents the ATVs dynamic models used in this paper. The design of the second order sliding mode observer and its convergence analysis are presented in Section 3. We present here the two cascaded observers designed to estimate the forces $F_{x}$ and the ground slope $\alpha$. Some results about the states estimations are presented is Section 4. The driving simulator SCANeR-studio, software developed by OKTAL (see www.oktal.fr) is used to integrate the G7.240 carrier dynamic model and test the driving performances. The data was acquired at a frequency of $20 \mathrm{~Hz}$. The proposed observers, to be tested, are integrated to run in real time in the driving simulator. Finally, some remarks and perspectives are given in the concluding section.

\section{Vehicle road interaction model}

\subsection{Background and G7 tractor model}

To develop a nominal model of the vehicle with uncertainties, we assume the car body to be rigid and that the pneumatic contacts are permanent and reduced to one point for each wheel.

Let us consider the fixed reference frame $R$ as basis and represent the vehicle by the scheme shown in Fig. 1 . The vehicle is composed of five sub-systems: the chassis translation, the chassis rotation, the suspensions elongations, the steering angle for the front wheels and the four wheels rotations. All these components lead to a sixteen degree of freedom (DOF) model.

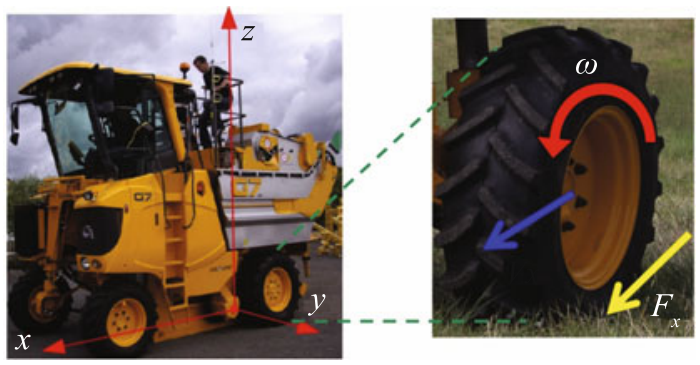

Fig. 1 Reference frame and G7 tractor model

$$
q=\left[x, y, z, \theta, \phi, \psi, z_{1}, z_{2}, z_{3}, z_{4}, \delta_{1}, \delta_{2}, \varphi_{1}, \varphi_{2}, \varphi_{3}, \varphi_{4}\right]^{\mathrm{T}} .
$$

The components of this vector are given by Table 1 .

Using a symbolic computation software and considering the generalized coordinate vector $q$, we can compute the Lagrange dynamic equations of the vehicle. The model is developed following the same approach as in [17]. Then the vehicle motion can be described by the following model:

$$
\begin{gathered}
\tau=M(q) \ddot{q}+C(q, \dot{q}) \dot{q}+V(q, \dot{q})+\eta_{o}(t, q, \dot{q}) \\
\tau=\Gamma_{e}+\Gamma=\Gamma_{e}+J^{\mathrm{T}} F .
\end{gathered}
$$

It can be shown that this model has several useful properties and that it corresponds to a passive system ${ }^{[18]}$.

The input torque $\tau$ is composed of a part produced by the engine and a part which can be assumed to be given by some feedback function and reaction of the ground ${ }^{[19,20]}$. The vectors $\dot{q}, \ddot{q} \in \mathbf{R}^{16}$ are velocities and corresponding accelerations, respectively. The 16-dimensional matrices $M(q)$ and
$C(q, \dot{q})$ are the inertia and the Coriolis and centrifugal forces matrices, respectively ${ }^{[17,18]}$ (see details in the Appendix).

Table 1 Nomenclature of the generalized coordinate vector

\begin{tabular}{cc}
\hline Variable & Definition \\
\hline$x$ & Longitudinal displacement of the vehicle \\
$y$ & Lateral displacement of the vehicle \\
$z$ & Vertical displacement of the vehicle \\
$\theta$ & Roll angle \\
$\phi$ & Pitch angle \\
$\psi$ & Yaw angle \\
$z_{1}, z_{2}$, & Vertical elongation for front right, \\
$z_{3}, z_{4}$ & front left, rear right and rear left suspension, respectively \\
$\delta_{1}, \delta_{2}$ & Steering angles for the two front wheels \\
$\varphi_{1}, \varphi_{2}$, & Rotation angle for front right, \\
$\varphi_{3}, \varphi_{4}$ & front left, rear right and rear left wheel, respectively \\
\hline
\end{tabular}

The vector $V(q, \dot{q})=\xi\left(K_{v} \dot{q}+K_{p} q\right)+G(q)$ is a 16 dimensional vector, the components of which summarize the suspensions and gravitation forces with $K_{v}$ and $K_{p}$ (the damping and the stiffness matrix, respectively).

$G(q)$ is the gravity term, and $\xi$ is equal to unity when the corresponding wheel is in contact with the ground and zero if not. $\eta_{o}(t, q, \dot{q})$ is the 16 -dimensional vector where its components represent the uncertainties and the neglected dynamics.

In (3), we remark that there are control inputs from driving $\Gamma_{e}$ and external inputs coming from exchanges with environment (road reactions) given by $J^{\mathrm{T}} F$ where $J$ is the Jacobian matrix, of dimensions $16 \times 12$, and $F$ is the 12 dimensional input force vector acting on the wheels (longitudinal $F_{x i}$, lateral $F_{y i}$ and normal $\left.F_{z i}, i=1, \cdots, 4\right)$ :

$$
\begin{aligned}
& F^{\mathrm{T}}= \\
& {\left[F_{x 1}, F_{y 1}, F_{z 1}, F_{x 2}, F_{y 2}, F_{z 2}, F_{x 3}, F_{y 3}, F_{z 3}, F_{x 4}, F_{y 4}, F_{z 4}\right] .}
\end{aligned}
$$

Then the vehicle dynamics can be described as a connection of five subsystems in a basic fixed frame of reference. These subsystems are chassis translation, chassis rotation, suspensions elongations, steering angle of the front wheels and wheels rotations. Every line of this equation represents a subsystem.

$$
\begin{aligned}
& {\left[\begin{array}{c}
F_{T} \\
F_{R} \\
F_{S} \\
\Gamma_{e 4} \\
\Gamma_{e 5}
\end{array}\right]=\left[\begin{array}{lllll}
\bar{M}_{11} & \bar{M}_{12} & \bar{M}_{13} & \underline{0}_{32} & \underline{0}_{34} \\
\bar{M}_{21} & \bar{M}_{22} & \bar{M}_{23} & \bar{M}_{24} & \bar{M}_{25} \\
\bar{M}_{31} & \bar{M}_{32} & \bar{M}_{33} & \underline{0}_{42} & \underline{0}_{44} \\
\underline{0}_{23} & \bar{M}_{42} & \underline{0}_{24} & \bar{M}_{44} & \underline{0}_{24} \\
\underline{0}_{43} & \bar{M}_{52} & \underline{0}_{44} & \underline{0}_{42} & \bar{M}_{55}
\end{array}\right]\left[\begin{array}{c}
\ddot{q}_{1} \\
\ddot{q}_{2} \\
\ddot{q}_{3} \\
\ddot{q}_{4} \\
\ddot{q}_{5}
\end{array}\right]+} \\
& {\left[\begin{array}{lllll}
\underline{0}_{33} & \bar{C}_{12} & \bar{C}_{13} & \underline{0}_{32} & \underline{0}_{34} \\
\underline{0}_{33} & \bar{C}_{22} & \bar{C}_{23} & \bar{C}_{24} & \bar{C}_{25} \\
\underline{0}_{43} & \bar{C}_{32} & \bar{C}_{33} & \underline{0}_{42} & \underline{0}_{44} \\
\underline{0}_{23} & \bar{C}_{42} & \underline{0}_{24} & \underline{0}_{22} & \bar{C}_{45} \\
\underline{0}_{43} & \bar{C}_{52} & \underline{0}_{44} & \bar{C}_{54} & \underline{0}_{44}
\end{array}\right]\left[\begin{array}{c}
\dot{q}_{1} \\
\dot{q}_{2} \\
\dot{q}_{3} \\
\dot{q}_{4} \\
\dot{q}_{5}
\end{array}\right]+\left[\begin{array}{c}
V_{1} \\
V_{2} \\
V_{3} \\
V_{4} \\
V_{5}
\end{array}\right]+\left[\begin{array}{l}
\eta_{1} \\
\eta_{2} \\
\eta_{3} \\
\eta_{4} \\
\eta_{5}
\end{array}\right] .}
\end{aligned}
$$

\subsection{Wheels contact models}

Sometimes, to develop some application in vehicle, we do not need to use all the global dynamic models of vehicle. It will be the case for estimation of the contact forces. Then 
from the last line of (4), the wheels dynamics of the ATV can be extracted in a partial model as

$$
\Gamma_{e 5}=\bar{M}_{52} \ddot{q}_{2}+\bar{M}_{55} \ddot{q}_{5}+\bar{C}_{52} \dot{q}_{2}+\bar{C}_{54} \dot{q}_{4}+V_{5}+\eta_{5}
$$

where

$$
\begin{aligned}
\Gamma_{e 5}= & {\left[\begin{array}{cc}
C_{m 1}-T_{1}-r F_{x 1} & 0 \\
0 & C_{m 2}-T_{2}-r F_{x 2} \\
0 & 0 \\
0 & 0 \\
0 & 0 \\
0 & 0 \\
C_{m 3}-T_{3}-r F_{x 3} & 0 \\
0 & C_{m 4}-T_{4}-r F_{x 4}
\end{array}\right] . }
\end{aligned}
$$

We obtain

$$
\ddot{q}_{5}=\bar{M}_{55}^{-1}\left[\Gamma_{e 5}-\bar{M}_{52} \ddot{q}_{2}-\bar{C}_{52} \dot{q}_{2}-\bar{C}_{54} \dot{q}_{4}-V_{5}-\eta_{5}\right] \text {. }
$$

Then the wheels dynamic system is given by the following simplified model

$$
\left\{\begin{array}{l}
\dot{\omega}_{1}=\frac{1}{J_{r}}\left(C_{m 1}-T_{1}-R_{e} F_{x 1}-\mu_{1}\right) \\
\dot{\omega}_{2}=\frac{1}{J_{r}}\left(C_{m 2}-T_{2}-R_{e} F_{x 2}-\mu_{2}\right) \\
\dot{\omega}_{3}=\frac{1}{J_{r}}\left(C_{m 3}-T_{3}-R_{e} F_{x 3}-\mu_{3}\right) \\
\dot{\omega}_{4}=\frac{1}{J_{r}}\left(C_{m 4}-T_{4}-R_{e} F_{x 4}-\mu_{4}\right)
\end{array}\right.
$$

where $\omega_{1}, \omega_{2}, \omega_{3}$ and $\omega_{4}$ are the angular velocity of the front left, front right, rear left and rear right wheel, respectively. $J_{r}$ is the inertia of tire. $R_{e}$ is the effective radius of the tire. $C_{m 1}, C_{m 2}, C_{m 3}$ and $C_{m 4}$ are the motor couple applied at the front left, front right, rear left and rear right wheel, respectively. $T_{1}, T_{2}, T_{3}$ and $T_{4}$ are the braking couple applied at the front left, front right, rear left and rear right wheel, respectively. $F_{x 1}, F_{x 2}, F_{x 3}$ and $F_{x 4}$ are the longitudinal force applied at the front left, front right, rear left and rear right wheel, respectively; $\mu_{i}$ (for $i=1, \cdots, 4$ ) are the coupling terms due to dynamics of the other subsystems and the neglected dynamics.

\section{State observer and structured esti- mation}

In this part we extend the observer of $[15,16]$ to enhance the robustness of the state estimation. We use the robust second order differentiator to build up an estimation scheme allowing ground slope estimation. The states are estimated in finite time. Then we estimate additionally the ground slope using only angular velocities measurements. The estimations are produced using two cascaded observersestimators. The diagram is represented in Fig. 2. The first block is a robust observer with unknown inputs and the second block develops a ground slope estimation.

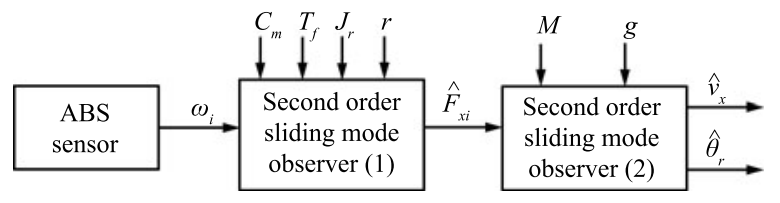

Fig. 2 Simulation block diagram

\subsection{Longitudinal tire forces observer}

\subsubsection{Unknown input observer design}

The first step produces state estimations. The longitudinal tire forces $F_{x i}$ (for $i=1, \cdots, 4$ ) are estimated in the second step. The robust differentiation observer is used for estimation of the angular accelerations of the wheels. The wheels angular velocities and the velocity of the vehicle body $v_{x}$ are assumed available for measurement.

Contact forces are the most difficult part to model in vehicle applications. Some assumptions are often used for existing models, for example, in the case of magic formula ${ }^{[21]}$ :

1) Assume wheel velocities are constant, then the slip and steering angles are constant.

2) Assume that the pneumatic stiffness $C_{x}$ and $C_{y}$ are invariant and constant.

3) Assume that the normal forces $F_{z}$ exerted on the wheels are constant.

4) Assume that the behavior is uniform.

Actual situation is different from steady state conditions considered when modeling tire contact forces for steady states experiments. This means that at least the road characteristics have some perturbations and variables have small random variation, perturbations or additional noise $e(t)$. In this work, without any knowledge on force characteristics, we consider the case of driving on a homogeneous straight road like on a highway. It can be justified if we get acceptable results and performance. We assume to have slow variations in average.

Let us consider that we have very small variations in the mean $\dot{F}_{x i} \simeq 0$. So the system (8) can be written in state space form as (for $i=1, \cdots, 4$ )

$$
\left\{\begin{array}{l}
\dot{\theta}_{i}=\omega_{i} \\
J_{r} \dot{\omega}_{i}=C_{m i}-T_{i}-R_{e} F_{x i}-\mu \\
\dot{F}_{x i} \simeq 0
\end{array}\right.
$$

where $\theta_{i}$ is the angular position of the corresponding wheel.

We chose the state vector $x_{1}=\theta_{i}, x_{2}=\omega_{i}$ and $x_{3}=F_{x i}$, the system (9) can be written in the state space form as

$$
\left\{\begin{array}{l}
\dot{x}_{1}=x_{2} \\
\dot{x}_{2}=\frac{1}{J_{r}}\left(C_{m i}-T_{i}-R_{e} x_{3}-\mu\right) \\
\dot{x}_{3}=0
\end{array}\right.
$$

We assume that the angular velocity $x_{2}$ is measured by the anti-lock brake systems (ABS) sensor. Then we used the second order sliding mode method to develop an observer to estimate the longitudinal tire forces $x_{3}$. This technique is an attractive approach for robustness ${ }^{[22,23]}$ and is able to reject perturbations and uncertainty effects ${ }^{[24]}$.

Then the observer is

$$
\left\{\begin{aligned}
\hat{\dot{x}}_{2}= & \frac{1}{J_{r}}\left(C_{m i}-T_{i}-R_{e} \hat{x}_{3}\right)+\Lambda_{1}\left|x_{2}-\hat{x}_{2}\right|^{\frac{1}{2}} \times \\
& \operatorname{sgn}\left(x_{2}-\hat{x}_{2}\right) \\
\hat{\dot{x}}_{3}= & \Lambda_{2} \operatorname{sgn}\left(x_{2}-\hat{x}_{2}\right)
\end{aligned}\right.
$$

where $\hat{x}_{2}$ and $\hat{x}_{3}$ are the state estimates of $x_{2}$ and $x_{3}$, re- 
spectively. $\Lambda_{1}$ and $\Lambda_{2}$ are positive gain matrices.

$$
\begin{aligned}
\Lambda_{1} & =\left[\begin{array}{cccc}
\lambda_{11} & 0 & 0 & 0 \\
0 & \lambda_{12} & 0 & 0 \\
0 & 0 & \lambda_{13} & 0 \\
0 & 0 & 0 & \lambda_{14}
\end{array}\right] \\
\Lambda_{2} & =\left[\begin{array}{cccc}
\lambda_{11} & 0 & 0 & 0 \\
0 & \lambda_{12} & 0 & 0 \\
0 & 0 & \lambda_{13} & 0 \\
0 & 0 & 0 & \lambda_{14}
\end{array}\right] .
\end{aligned}
$$

This matrices are chosen such that the dynamics of the observation error converge to zero.

\subsubsection{Convergence analysis}

Let $\tilde{x}_{2}=x_{2}-\hat{x}_{2}$ and $\tilde{x}_{3}=x_{3}-\hat{x}_{3}$ be the state estimations error. Then the observation error dynamics is

$$
\left\{\begin{array}{l}
\dot{\tilde{x}}_{2}=-\frac{R_{e}}{J_{r}} \tilde{x}_{3}-\Lambda_{1}\left|\tilde{x}_{2}\right|^{\frac{1}{2}} \operatorname{sgn}\left(\tilde{x}_{2}\right) \\
\dot{\tilde{x}}_{3}=-\Lambda_{2} \operatorname{sgn}\left(\tilde{x}_{2}\right) .
\end{array}\right.
$$

The convergence analysis is based on Lyapunov approach to describe the averaged behaviour ${ }^{[23,24]}$. In (12), averaging behaviour analysis is considered.

Step 1. The convergence of $x_{3}$.

The Lyapunov candidate function $V_{1}=\frac{1}{2} \tilde{x}_{3}^{\mathrm{T}} \tilde{x}_{3}$, shows that $\tilde{x}_{2}=0$ is an attractive surface if $V_{1}<0$ :

$$
\dot{V}_{1}=\tilde{x}_{3}^{\mathrm{T}}\left(-\Lambda_{2} \operatorname{sgn}\left(\tilde{x}_{2}\right)\right) .
$$

Then we choose $\lambda_{2 i}$ as

$$
\lambda_{2 i}>\left|\tilde{x}_{2 i}\right|, \quad i=1, \cdots, 4 .
$$

The convergence in finite time $t_{0}$ for the system state is obtained: $\hat{x}_{2}$ goes to $x_{2}$ in finite time $t_{0}$, so in the average $\tilde{x}_{2}=0, \forall t>t_{0}$.

Remark 1. It is important to note that, the convergence is considered in the mean average (e.g., the PWM signals for motor control). So it is ensured that the mean average state value goes to zero in finite time $t_{0}$. In practice, the actual system is low pass. Then we can avoid additional low pass filtering of the estimated value.

Step 2. The convergence of $x_{2}$.

For $t>t_{0}$, we have in the mean average $\tilde{x}_{3}=0$, we can then deduce from (12) that

$$
\dot{\tilde{x}}_{2}=-\Lambda_{1}\left|\tilde{x}_{2}\right|^{\frac{1}{2}} \operatorname{sgn}\left(\tilde{x}_{2}\right) .
$$

Let a second Lyapunov candidate function $V_{2}=\frac{1}{2} \tilde{x}_{2}^{\mathrm{T}} \tilde{x}_{2}$. The derivative of this function is

$$
\dot{V}_{2}=\tilde{x}_{2}^{\mathrm{T}}\left(-\Lambda_{1}\left|\tilde{x}_{2}\right|^{\frac{1}{2}} \operatorname{sgn}\left(\tilde{x}_{2}\right)\right) .
$$

To ensure that $V_{2}<0$ and then $\hat{x}_{2}$ converges to $x_{2}$ in the mean average (see previous remark), we must choose $\Lambda_{1}$ as

$$
\lambda_{1 i}>\left|\tilde{x}_{2 i}\right|, \quad i=1, \cdots, 4 .
$$

In conclusion, the condition to get the convergence of our observer can be summarized as

$$
\lambda_{2 i}>\left|\tilde{x}_{2 i}\right|, \quad \lambda_{1 i}>\left|\tilde{x}_{2 i}\right|, \quad \text { for } i=1, \cdots, 4 .
$$

The convergence of the state $x_{2}$ and $x_{3}$ has been proved. The observation errors go to a neighborhood of zero in finite time. The error bound depends on the chosen gain parameters $\lambda$.

\subsection{Ground slope estimator}

The ground has a more complex behavior. Then in this part, the longitudinal tire forces estimations are used to build up the ground slope and the longitudinal velocity of vehicle. This robust observer is also based on a second order sliding mode observer.

The estimation of the road slope is achieved on the basis of a longitudinal model given by (18). The longitudinal tire forces $\hat{F}_{x i}$ obtained with the first observer can thus be used as an input for the longitudinal model. The slope ground is presented in Fig. 3 .

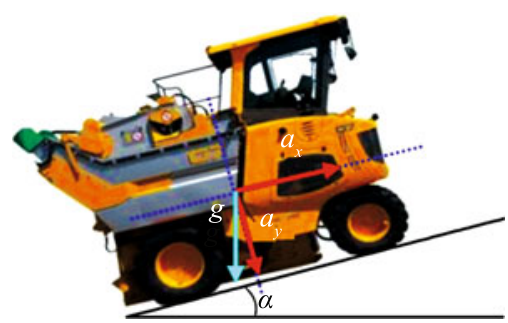

Fig. 3 ATV in a slope angle

Then we consider the following model

$$
\left\{\begin{array}{l}
\dot{v}_{x}=\frac{\sum_{i=1}^{4} F_{x i}}{M}-g \sin \alpha \\
\alpha \simeq 0
\end{array}\right.
$$

where $v_{x}$ is the longitudinal velocity, $M$ is the mass of body, $\alpha$ is the road slope angle. $F_{x 1}, F_{x 2}, F_{x 3}$ and $F_{x 4}$ are the longitudinal forces applied at the front left, front right, rear left and rear right wheel, respectively.

We considered that the road does not present bumps. Then the slope angle variation is zero. Generally, this angle is less than ten degrees, so one can approximate $\sin \alpha$ by $\alpha$.

Thus, the system (18) can be written in the following form as

$$
\underline{\dot{x}}=\underline{A} \underline{x}+\underline{B} \underline{u}
$$

where, $\underline{x}=\left[\underline{x}_{1} \underline{x}_{2}\right]=\left[v_{x} \alpha\right]$ is the state vector, $\underline{u}=\sum_{i=1}^{4} \hat{F}_{x i}$ is the control input, $\underline{C}=\left[\begin{array}{ll}1 & 0\end{array}\right]$ the matrix output, while matrices $\underline{A}$ and $\underline{B}$ have the following forms

$$
\underline{A}=\left[\begin{array}{cc}
0 & -g \\
0 & 0
\end{array}\right], \quad \underline{B}=\left[\begin{array}{c}
\frac{1}{M} \\
0
\end{array}\right] .
$$

We can easily verify that the rank of the observability matrix is two. Then the system is obviously observable.

In order to estimate the road slope angle, a second order sliding observer is chosen, which is given by

$$
\underline{\dot{\hat{x}}}=\underline{A} \underline{\hat{x}}+\underline{B} \underline{\hat{u}}+S
$$


where $\underline{\hat{x}}$ and $\underline{\hat{u}}$ are the estimation of $\underline{x}$ and $\underline{u}$, respectively. $S$ is the observation term

$$
S=\left[\begin{array}{c}
\underline{\Lambda}_{1}\left|\underline{x}_{1}-\underline{\hat{x}}_{1}\right|^{\frac{1}{2}} \operatorname{sgn}\left(\underline{x}_{1}-\underline{\hat{x}}_{1}\right) \\
\underline{\Lambda}_{2} \operatorname{sgn}\left(\underline{x}_{1}-\underline{\hat{x}}_{1}\right)
\end{array}\right]
$$

where $\underline{\Lambda}_{1}$ and $\underline{\Lambda}_{2}$ are the observer gains calculated by convergence analysis. Then we use the Lyapunov candidate function to prove the convergence of this observer.

\section{Experimental set up and application}

In this section, we give some results in order to test and validate our approach on the proposed observers. We use the driving simulator of vehicle $\mathrm{SCANeR}^{\mathrm{TM}}$-Studio which is in laboratory $\operatorname{LSIS}^{3}$.

\subsection{Experimental set up $\mathrm{SCANeR}^{\mathrm{TM}}$ studio driving simulator}

The SCANeR ${ }^{\mathrm{TM}}$-Studio is a driving simulator which uses specific softwares to enhance the virtual driving environment and immerse drivers in completely realistic traffic situations. The SCANeR ${ }^{\mathrm{TM}}$-Studio features (see Fig. 4) are regrouped according to different components to run experimentation: $>$ Core; D Driver; $\$ Environment; V Vehicle; - Simulation.

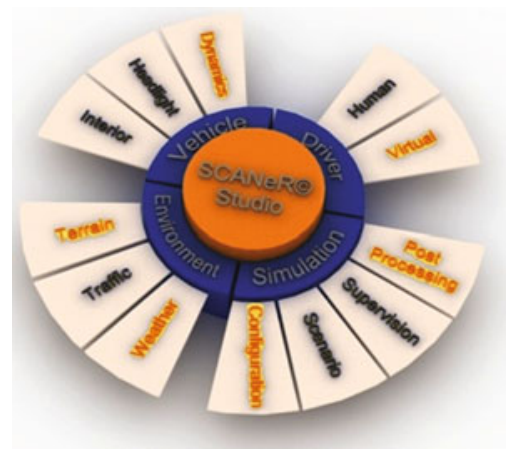

Fig. 4 SCANeR TM $^{\mathrm{TM}}$ Studio features

$\mathrm{SCANeR}^{\mathrm{TM}}$-Studio is a professional driving simulator proposed by $\mathrm{Oktal}^{4}$. This driving simulator can be used for prototyping and for designing a function list of vehicle. It will help us to evaluate performance of our observers.

\subsection{The G7 in scanerstudio environment simulator}

Our laboratory and namely the staff SASV is involved in the ActiSurTT research project funded by the French National Agency for Research (ANR: Agence Nationale de la Recherche). Our objective is modeling, designing observers and diagnosis to enhance the safety of all terrain vehicle. In the framework of this project, a grape harvesting machine G7 (see Fig. 5) from the Gregoire company has been chosen as the experimental target for testing the active systems safety based on our robust observers. The first aim achieved was the use of the different analytical models that we have proposed in SCANeR ${ }^{\mathrm{TM}}$-Studio environment. This

\footnotetext{
${ }^{3}$ www.lsis.org

${ }^{4}$ www.oktal.fr
}

step needed to adapt the tools used in SCANeR ${ }^{\mathrm{TM}}$-Studio with those used from other modeling environment. So we have introduced different analytically models (geometric, kinematics and dynamic models) in the SCANeR ${ }^{\mathrm{TM}}$-Studio environment.

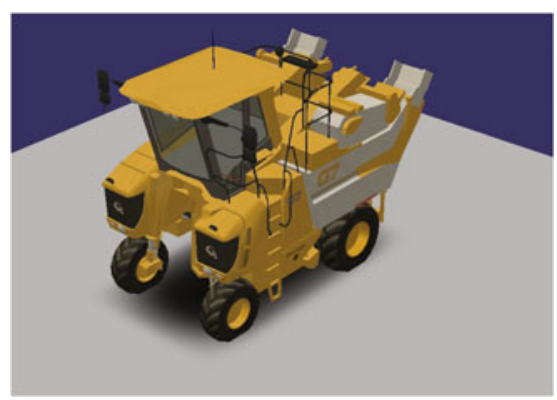

Fig. 5 Gregoire vehicle G7

For these purposes, SCANeR ${ }^{\mathrm{TM}}$-Studio has been used to provide a realistic environment for testing and validating different theoretical approaches. For the test, we started with the definition of the region used for experimental tests for validation and its integration to $\mathrm{SCANeR}^{\mathrm{TM}}-$ Studio (like defined by Google Map with additional information on the ground characteristics).

So in the first step Google sketchup software was used to generate realistic 3D (geometric) simulation environments files that accurately describe the field on which $\mathrm{G} 7$ did all the actual manoeuvres corresponding to specific planned scenarios. This environment provides a realistic model that could be used for any kinds of paths provided that dynamic and kinetic characteristics of the ground are included, as we did. When driving the G7, any path can be followed without needing for instance the GPS data, we can re-simulate the GPS defined trajectory really followed in the experiments. These are used for the validations.

Finally all observers, estimators and diagnosis tools can be tested in a simple way (from analytical equations using Matlab/Simulink) on the behavioral models developed on Scanner Studio exactly as it was the actual G7.240 carrier.

\subsection{Results}

In the simulation, the state and tire forces are generated by the G7 tractor with the car driving simulator SCANeR ${ }^{\mathrm{TM}}$-Studio. The driving simulation was generated by the SCANeR-Studio software (OKTAL, France) and driving performance data was recorded at a frequency of $20 \mathrm{~Hz}$. Fig. 6 presents the trajectory used for this simulation. The ATVs speed variations is presented in Fig. 7. We remark that the speed of G7 tractor is almost constant at approximately $9 \mathrm{~km} / \mathrm{h}$.

Fig. 8 shows the measurements angular velocities of the four wheels. These values are used as input in our first observer to estimate the longitudinal tire forces. These estimations are presented by Fig. 9. We show a good asymptotic convergence in finite time. Fig. 10 presents the real and the estimation of the ground slope. We also remark that the performance of this estimation approach is very satisfactory since the estimation error is minimal. 


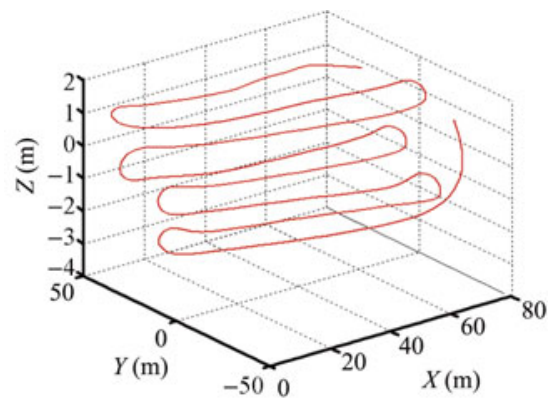

Fig. 6 The trajectory used in our simulation

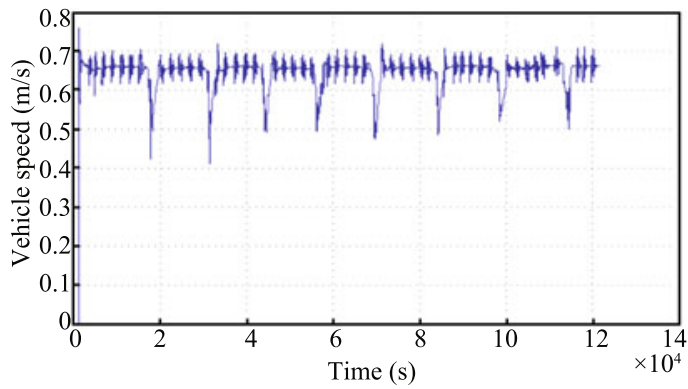

Fig. 7 The ATVs speed $(\mathrm{m} / \mathrm{s})$
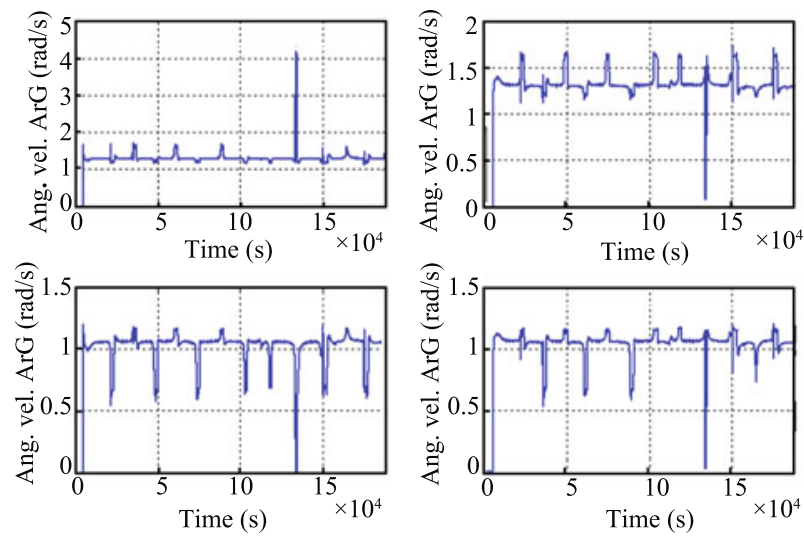

Fig. 8 The angular velocities (Ang.: angular, vel.:velocity)
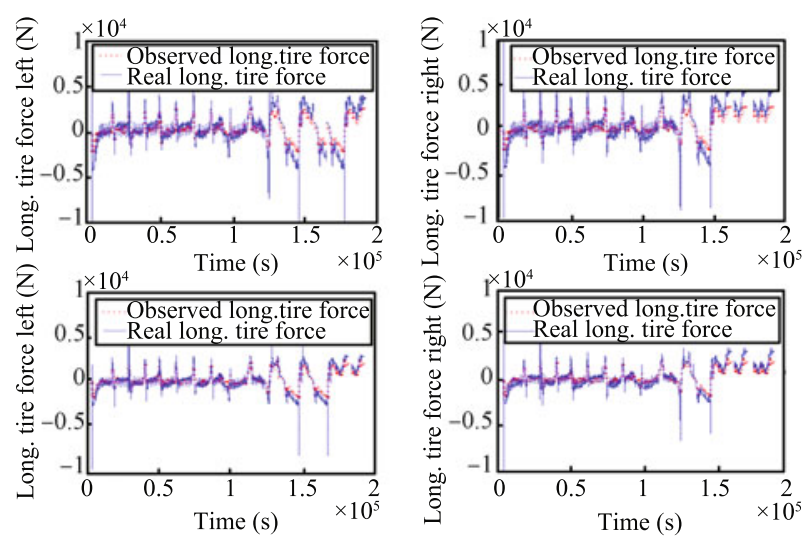

Fig. 9 Longitudinal tire forces (Long.: longitudinal)

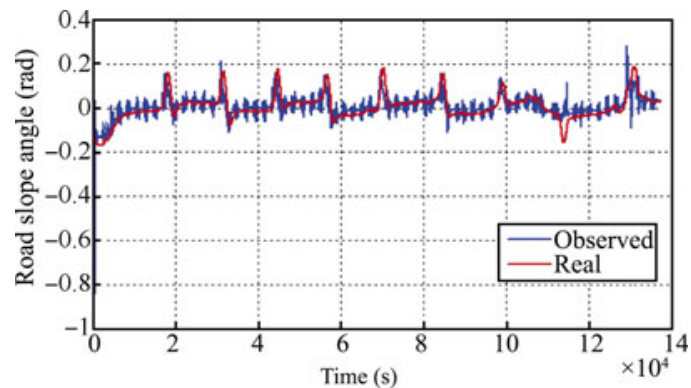

Fig. 10 The ground slope

\section{Conclusions}

In this paper, we present, as a first block, an observer to estimate the vehicle state variables, in first step, and the longitudinal tire forces for all-terrain vehicle in a second step. The proposed observer is based on second order sliding mode. In the second block, these estimations are used as input for a second observer to estimate the longitudinal velocity and the ground slope. The finite time convergence of the state observer is proved. The performances of this observer are validated by the experimental results and use of a driving simulator. We used the professional driving simulator $\mathrm{SCANeR}^{\mathrm{TM}}$-Studio. The actual results prove effectiveness of the proposed method for all-terrain heavy vehicles on (with a deformable and unstructured ground). This is mainly due to the robustness of the second order sliding mode observers which allow better rejection of perturbation and then better reconstruction of the unknown inputs.

In our future investigations, we estimate the lateral tire forces and the ground banking angle for ATVs. After that, all these estimations (longitudinal and lateral tire forces, ground slope and road bank angle) can be used to generate an alarm system for rollover situation for all-terrain vehicles.

\section{Acknowledgement}

The authors are grateful to the Agence Nationale de la Recherche (ANR) and to the ActiSurTT project partners. This project is funded by the French National Research Agency.

Many thanks also to Oktal for the assistance to integrate the model of the ground contact simulation for G7.240 in $\mathrm{SCANeR}^{\mathrm{TM}}$-Studio.

\section{Appendix (Parameters of the models)}

The inertia matrix $M$ and the reduced inertia matrices $M_{i j}$ :

$$
M=\left[\begin{array}{ccccc}
\bar{M}_{11} & \bar{M}_{12} & \bar{M}_{13} & \underline{0}_{32} & \underline{0}_{34} \\
\bar{M}_{21} & \bar{M}_{22} & \bar{M}_{23} & \bar{M}_{24} & \bar{M}_{25} \\
\bar{M}_{31} & \bar{M}_{32} & M_{33} & \underline{0}_{42} & \underline{0}_{44} \\
\underline{0}_{23} & \bar{M}_{42} & \underline{0}_{24} & \bar{M}_{44} & \underline{0}_{24} \\
\underline{0}_{43} & \bar{M}_{52} & \underline{0}_{44} & \underline{0}_{42} & \bar{M}_{55}
\end{array}\right]
$$




$$
\begin{aligned}
& \bar{M}_{33}=\left[\begin{array}{cccc}
M_{7}^{7} & 0 & 0 & 0 \\
0 & M_{8}^{8} & 0 & 0 \\
0 & 0 & M_{9}^{9} & 0 \\
0 & 0 & 0 & M_{10}^{10}
\end{array}\right] \\
& \bar{M}_{44}=\left[\begin{array}{cc}
M_{11}^{11} & 0 \\
0 & M_{12}^{12}
\end{array}\right] \\
& \bar{M}_{12}=\bar{M}_{21}^{\mathrm{T}}=\left[\begin{array}{ccc}
M_{1}^{4} & M_{1}^{5} & M_{1}^{6} \\
M_{2}^{4} & M_{2}^{5} & M_{2}^{6} \\
0 & M_{3}^{5} & M_{3}^{5}
\end{array}\right] \\
& \bar{M}_{55}=\left[\begin{array}{cccc}
J_{r 1} & 0 & 0 & 0 \\
0 & J_{r 2} & 0 & 0 \\
0 & 0 & J_{r 3} & 0 \\
0 & 0 & 0 & J_{r 4}
\end{array}\right] \\
& \bar{M}_{13}=\bar{M}_{31}^{\mathrm{T}}=\left[\begin{array}{cccc}
M_{1}^{7} & M_{1}^{8} & M_{1}^{9} & M_{1}^{10} \\
M_{2}^{7} & M_{2}^{8} & M_{2}^{9} & M_{2}^{10} \\
M_{3}^{7} & M_{3}^{8} & M_{3}^{9} & M_{3}^{10}
\end{array}\right] \\
& \bar{M}_{11}=\left[\begin{array}{ccc}
M_{1}^{1} & 0 & 0 \\
0 & M_{2}^{2} & 0 \\
0 & 0 & M_{3}^{3}
\end{array}\right] \\
& \bar{M}_{24}=\bar{M}_{42}^{\mathrm{T}}=\left[\begin{array}{cc}
M_{4}^{11} & M_{4}^{12} \\
M_{5}^{11} & M_{5}^{12} \\
0 & 0
\end{array}\right] \\
& \bar{M}_{22}=\left[\begin{array}{ccc}
M_{4}^{4} & M_{4}^{5} & M_{4}^{6} \\
M_{5}^{4} & M_{5}^{5} & M_{5}^{6} \\
M_{6}^{4} & M_{6}^{5} & M_{6}^{6}
\end{array}\right] \\
& \bar{M}_{23}=\bar{M}_{32}^{\mathrm{T}}=\left[\begin{array}{llll}
M_{4}^{7} & M_{4}^{8} & M_{4}^{9} & M_{4}^{10} \\
M_{5}^{7} & M_{5}^{8} & M_{5}^{9} & M_{5}^{10} \\
M_{6}^{7} & M_{6}^{8} & M_{6}^{9} & M_{6}^{10}
\end{array}\right] \\
& \bar{M}_{25}=\bar{M}_{52}^{\mathrm{T}}=\left[\begin{array}{cccc}
M_{4}^{13} & M_{4}^{14} & M_{4}^{15} & M_{4}^{16} \\
M_{5}^{13} & M_{5}^{14} & M_{5}^{15} & M_{5}^{16} \\
0 & 0 & M_{6}^{15} & M_{6}^{16}
\end{array}\right] .
\end{aligned}
$$

Coriolis and centrifugal matrix $C$ and the reduced Coriolis and centrifugal matrices $C_{i j}$ :

$$
\begin{gathered}
C=\left[\begin{array}{lllll}
\underline{0}_{33} & \bar{C}_{12} & \bar{C}_{13} & \underline{0}_{32} & \underline{0}_{34} \\
\underline{0}_{33} & \bar{C}_{22} & \bar{C}_{23} & \bar{C}_{24} & \bar{C}_{25} \\
\underline{0}_{43} & \bar{C}_{32} & \underline{0}_{44} & \underline{0}_{42} & \underline{0}_{44} \\
\underline{0}_{23} & \bar{C}_{42} & \underline{0}_{24} & \underline{0}_{22} & \bar{C}_{45} \\
\underline{0}_{43} & \bar{C}_{52} & \underline{0}_{44} & \bar{C}_{54} & \underline{0}_{44}
\end{array}\right] \\
\bar{C}_{45}=\bar{C}_{54}^{\mathrm{T}}=\left[\begin{array}{cccc}
0 & 0 & C_{11}^{15} & 0 \\
0 & 0 & 0 & C_{12}^{16}
\end{array}\right] \\
\bar{C}_{23}=\bar{C}_{32}^{\mathrm{T}}=\left[\begin{array}{cccc}
C_{4}^{7} & C_{4}^{8} & C_{4}^{9} & C_{4}^{10} \\
C_{5}^{7} & C_{5}^{8} & C_{5}^{9} & C_{5}^{10} \\
C_{6}^{7} & C_{6}^{8} & C_{6}^{9} & C_{6}^{10}
\end{array}\right] \\
\bar{C}_{13}=\left[\begin{array}{ccccc}
C_{1}^{7} & C_{1}^{8} & C_{1}^{9} & C_{1}^{10} \\
C_{2}^{7} & C_{2}^{8} & C_{2}^{9} & C_{2}^{10} \\
C_{3}^{7} & C_{3}^{8} & C_{3}^{9} & C_{3}^{10}
\end{array}\right]
\end{gathered}
$$

$$
\begin{aligned}
& \bar{C}_{24}=\bar{C}_{42}^{\mathrm{T}}=\left[\begin{array}{cc}
C_{4}^{11} & C_{4}^{12} \\
C_{5}^{11} & C_{5}^{12} \\
C_{6}^{11} & C_{6}^{12}
\end{array}\right] \\
& \bar{C}_{12}=\left[\begin{array}{ccc}
C_{1}^{4} & C_{1}^{5} & C_{1}^{6} \\
C_{2}^{4} & C_{2}^{5} & C_{2}^{6} \\
0 & C_{3}^{5} & C_{3}^{6}
\end{array}\right] \\
& \bar{C}_{25}=\bar{C}_{52}^{\mathrm{T}}=\left[\begin{array}{cccc}
C_{4}^{13} & C_{4}^{14} & C_{4}^{15} & C_{4}^{16} \\
C_{5}^{13} & C_{5}^{14} & C_{5}^{15} & C_{5}^{16} \\
C_{6}^{13} & C_{6}^{14} & C_{6}^{15} & C_{6}^{16}
\end{array}\right] \\
& \bar{C}_{22}=\left[\begin{array}{ccc}
C_{4}^{4} & C_{4}^{5} & C_{4}^{6} \\
C_{5}^{4} & C_{5}^{5} & C_{5}^{6} \\
C_{6}^{4} & C_{6}^{5} & C_{6}^{6}
\end{array}\right] .
\end{aligned}
$$

The Jacobian matrix $J$ and the reduced Jocobian matrices $J_{i}$ :

$$
\begin{aligned}
& J=\left[\begin{array}{lllll}
J_{1}^{\mathrm{T}} & J_{2}^{\mathrm{T}} & J_{3}^{\mathrm{T}} & \underline{0}_{2,12} & \underline{0}_{4,12}
\end{array}\right]_{16 \times 12}^{\mathrm{T}}, \\
& J_{1}=\left[\begin{array}{ccc}
J_{1}^{1} & 0 & 0 \\
0 & J_{2}^{2} & 0 \\
0 & 0 & J_{3}^{3} \\
J_{4}^{1} & 0 & 0 \\
0 & J_{5}^{2} & 0 \\
0 & 0 & J_{6}^{3} \\
J_{7}^{1} & 0 & 0 \\
0 & J_{8}^{2} & 0 \\
0 & 0 & J_{9}^{3} \\
J_{10}^{1} & 0 & 0 \\
0 & J_{11}^{2} & 0 \\
0 & 0 & J_{12}^{3}
\end{array}\right], \quad J_{2}=\left[\begin{array}{ccc}
J_{1}^{4} & J_{1}^{5} & J_{1}^{6} \\
J_{2}^{4} & J_{2}^{5} & J_{2}^{6} \\
0 & J_{3}^{5} & J_{3}^{6} \\
J_{4}^{4} & J_{4}^{5} & J_{4}^{6} \\
J_{5}^{4} & J_{5}^{5} & J_{5}^{6} \\
0 & J_{6}^{5} & J_{6}^{6} \\
J_{7}^{4} & J_{7}^{5} & J_{7}^{6} \\
J_{8}^{4} & J_{8}^{5} & J_{8}^{6} \\
0 & J_{9}^{5} & J_{9}^{6} \\
J_{10}^{4} & J_{10}^{5} & J_{10}^{6} \\
J_{11}^{4} & J_{11}^{5} & J_{11}^{6} \\
0 & J_{12}^{5} & J_{12}^{6}
\end{array}\right], \\
& J_{3}=\left[\begin{array}{cccc}
J_{1}^{7} & 0 & 0 & 0 \\
J_{2}^{7} & 0 & 0 & 0 \\
J_{3}^{7} & 0 & 0 & 0 \\
0 & J_{4}^{8} & 0 & 0 \\
0 & J_{5}^{8} & 0 & 0 \\
0 & J_{6}^{8} & 0 & 0 \\
0 & 0 & J_{7}^{9} & 0 \\
0 & 0 & J_{8}^{9} & 0 \\
0 & 0 & J_{9}^{9} & 0 \\
0 & 0 & 0 & J_{10}^{10} \\
0 & 0 & 0 & J_{11}^{10} \\
0 & 0 & 0 & J_{12}^{10}
\end{array}\right]
\end{aligned}
$$

\section{References}

[1] M. A. Brandenburg, S. J. Brown, P. Archer, E. N. Brandt. All-terrain vehicle crash factors and associated injuries in patients presenting to a regional trauma center. The Journal of Trauma: Injury, Infection, and Critical Care, vol. 63, no. 5, pp. 994-999, 2007.

[2] E. Yonca. Modeling Severe ATV Injuries Using Artificial Neural Networks, Ph. D. dissertation, University of Ottawa, Canada, 2005. 
[3] E. Ono, K. Asano, M. Sugai, S. Ito, M. Yamamoto, M. Sawada, Y. Yasui. Estimation of automotive tire force characteristics using wheel velocity. Control Engineering Practice, vol. 11, no. 12, pp. 1361-1370, 2003.

[4] N. M'Sirdi, A. Rabhi, L. Fridman, J. Davila, Y. Delanne. Second order sliding mode observer for estimation of velocities, wheel sleep, radius and stiffness. In Proceedings of American Control Conference, IEEE, Minnesota, USA, pp. 3316-3321, 2006.

[5] U. Kiencke, L. Nielsen. Automotive Control System, Berlin, Heidelberg, New York: Springer, 2000.

[6] L. Ray. Nonlinear tire force estimation and road friction identification: Simulation and experiments. Automatica, vol. 33, no. 10, pp. 1819-1833, 1997.

[7] L. Ray. Nonlinear state and tire force estimation for advanced vehicle control. IEEE Transactions on Control Systems Technology, vol. 3, no. 1, pp. 117-124, 1999.

[8] J. Villagra, B. D'Andrea Novel, M. Fliess, H. Mounier. Estimation of longitudinal and lateral vehicle velocities: An algebraic approach. In Proceedings of the American Control Conference, IEEE, Seattle, WA, USA, pp. 3941-3946, 2008.

[9] F. Gustafsson. Slip-based tire-road friction estimation. Automatica, vol. 33, no. 6, pp. 1087-1097, 1997.

[10] A. Ungoren, H. Peng, H. E. Tseng. Experimental verification of lateral speed estimation methods. In Proceedings of the 6th International Symposium on Advanced Vehicle Control (AVEC 2002), Hiroshima, Japan, pp. 361-366, 2002.

[11] C. Carlson. Estimation with Applications for Automobile Dead Reckoning and Control, Ph. D. dissertation, University of Standford, USA, 2004.

[12] K. Mohamed, M. Chadli, M. Chaabane. Unknown inputs observer for a class of nonlinear uncertain systems: An LMI approach. International Journal of Automation and Computing, vol. 9, no. 3, pp. 331-336, 2012.

[13] O. M. Mohamed Vall, R. M'hiri. An approach to polynomial NARX/NARMAX systems identification in a closedloop with variable structure control. International Journal of Automation and Computing, vol. 5, no. 3, pp.313-318, 2008.

[14] J. Chen, E. Prempain, Q. H. Wu. Observer-based nonlinear control of a torque motor with perturbation estimation. International Journal of Automation and Computing, vol.3, no. 1, pp. 84-90, 2006.

[15] B. Jaballah, N. M'Sirdi, A. Naamane, H. Messaoud. Robust partial states observers for unknown inputs estimation in vehicle dynamics. International Journal of Research and Reviews in Computer Science, vol. 2, no. 2, pp. 604-611, 2011.

[16] B. Jaballah, N. K. M'Sirdi, A. Naamane, H. Messaoud. Estimation of vehicle longitudinal tire force with FOSMC and SOSMC. International Journal of Sciences and Technique of Automatic Control and Computer Engineering, vol. 5, no. 1, pp. 1516-1531, 2011.

[17] N. K. M'Sirdi, A. Rabhi, A. Naamane. Vehicle models and estimation of contact forces and tire road friction. In Proceedings of the 4th International Conference on Informatics in Control, Automation and Robotics (ICINCO), Angers, France, pp. 351-358, 2007.
[18] N. M'Sirdi, B. Jaballah, A. Naamane, H. Messaoud. Robust observers and unknown input observers for estimation, diagnosis and control of vehicle dynamics. In Proceedings of IEEE International Conference on Intelligent Robots and Systems (IROS08), IEEE, Nice, France, pp. 48-57, 2008.

[19] C. L. Clover, J. E. Bernard. Longitudinal tire dynamics. Vehicle System Dynamics: International Journal of Vehicle Mechanics and Mobility, vol. 29, no. 4, pp. 231-259, 1998.

[20] P. F. H. Dugoff, L. Segel. An analysis of tire traction properties and their influence on vehicle dynamic performance. SAE Transactions, vol. 3, pp. 1219-1243, 1970.

[21] H. B. Pacejka, I. J. M. Besseling. Magic formula tyre model with transient properties. Vehicle System Dynamics: International Journal of Vehicle Mechanics and Mobility, vol. 27, no. 1, pp. 234-249, 1997.

[22] J. Davila, L. Fridman, A. Levant. Second order sliding mode observer for mechanical systems. IEEE Transactions on $\mathrm{Au}-$ tomatic Control, vol. 50, no. 11, pp. 1785-1789, 2005.

[23] L. Fridman, A. Leavnt, J. Davila. High-order sliding-mode observation and identification for linear systems with unknown inputs. In Proceedings of the 45th IEEE Conference on Decision in Control, IEEE, San Diego, CA, USA, pp. 5567-5572, 2006.

[24] V. Utkin, J. Guldner, J. Shi. Sliding Mode Control in Electromechanical Systems, London, UK: Taylor Francis, 1999.

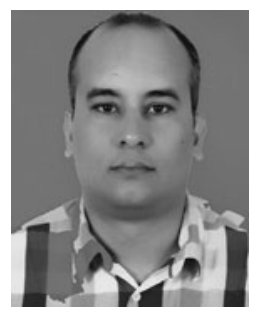

Belgacem Jaballah received his B.Sc. degree in electrical engineering from High School of Technical Education (ESSTT) in Tunis, Tunisia in 2003, his M. Sc. degree in automatic and industrial maintenance from School of Engineers of Monastir, Tunisia (ENIM) in 2006, and his Ph. D. degree in control engineering from University of AixMarseille, France and from University of Monastir, Tunisia in 2011

His research interests include adaptive and robust control, diagnosis and robust observation and control techniques for complex nonlinear systems such as in-vehicle dynamics.

E-mail: belgacem.jaballah@lsis.org

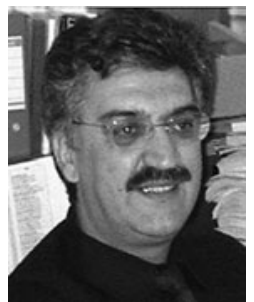

Nacer Kouider M'Sirdi received his $\mathrm{Ph} . \mathrm{D}$. degree in electronics (1983) and doctorat dEtat (1988) from the INPG of Grenoble. He was assistant professor in University of Paris VI (1987) and professor at the University of Versailles (1993) and dean of (LRV) Robotics Laboratory of Versailles. He is a professor at Polytech Marseille, Aix-Marseille University. Since 2005, he has been a research member of the LSIS (UMR CNRS 7296). In 2009, he created the variable structure automated systems (VSAS) research project on variable structure automatic systems (SASV) for fundamental research in automatic control and optimization of variable structure systems with commutations.

His research interests include adaptive and robust control, signal processing, diagnosis and observation for complex systems such as vehicles and robots.

E-mail: nacer.msirdi@lsis.org (Corresponding author) 